\title{
Structural Abnormalities of the Coronary Arterial Wall-in Addition to Luminal Narrowing-Affect Myocardial Blood Flow Reserve
}

\author{
Riccardo Liga ${ }^{1}$, Cecilia Marini ${ }^{2}$, Michele Coceani ${ }^{3}$, Elena Filidei ${ }^{3}$, Mathis Schlueter ${ }^{3}$, Massimiliano Bianchi ${ }^{4}$, \\ Giuseppe Rossi ${ }^{3,4}$, Silvia Pardini ${ }^{4}$, Piero Salvadori ${ }^{4}$, Oberdan Parodi ${ }^{4}$, Daniele Rovai ${ }^{3,4}$, Gianmario Sambuceti ${ }^{5}$, \\ Paolo Marraccini ${ }^{3,4}$, and Danilo Neglia ${ }^{3,4}$ \\ ${ }^{1}$ Scuola Superiore Sant'Anna, Pisa, Italy; ${ }^{2}$ CNR Institute of Bioimages and Molecular Physiology, Milan, Section of Genova, Italy; \\ ${ }^{3}$ Fondazione Toscana G. Monasterio, Pisa, Italy; ${ }^{4}$ CNR, Institute of Clinical Physiology, Pisa, Italy; and ${ }^{5}$ University of Genova, \\ Genova, Italy
}

\begin{abstract}
Multislice CT provides information on coronary luminal narrowing and on the structural abnormalities of the coronary arterial wall using densitometric analysis. We sought to investigate the effects of coronary luminal narrowing, structural abnormalities of the coronary arterial wall, and cardiovascular risk factors on regional and global myocardial blood flow (MBF) reserve. Methods: We studied 68 patients (mean age $\pm \mathrm{SD}$, $61 \pm 10 \mathrm{y} ; 41$ men, 27 women) with an intermediate probability of coronary artery disease. We measured the severity of coronary stenoses and the fibroadipose, fibromuscular, and calcium components of the coronary arterial wall by 64-row multislice CT coronary angiography. We also measured regional and global MBF reserve by PET using ${ }^{13} \mathrm{~N}-$ ammonia as a flow tracer at rest and after dipyridamole. Results: One or more significant coronary stenoses ( $\geq 50 \%$ luminal narrowing) was present in 32 patients (47\%), and nonsignificant stenoses were present in 15 patients $(22 \%)$. Regional MBF reserve was significantly different in the territories perfused by normal coronary arteries, nonsignificant coronary stenoses, and significant coronary stenoses $(P<0.001)$. Calcium content was higher in the coronary arteries with significant or nonsignificant stenoses $(0.95 \% \pm 1.08 \%$ and $0.73 \% \pm 0.93 \%$, respectively) than in those without stenoses $(0.11 \% \pm 0.38 \%$, $P<0.001)$. Significant coronary stenosis $(P=0.047)$ and calcium content $(P=0.017)$ were the only independent determinants of impaired regional MBF reserve using multivariate analysis. At multiple logistic regression analysis, the Framingham risk score, an index of global cardiovascular risk burden, was the only significant determinant of global MBF reserve $(P=0.028)$. Conclusion: Coronary stenoses and coronary calcium content independently affect regional MBF reserve. Framingham risk score is the only significant determinant of global MBF reserve.
\end{abstract}

\footnotetext{
Received Mar. 26, 2011; revision accepted Jun. 13, 2011.

For correspondence or reprints contact: Danilo Neglia, Fondazione Toscana G. Monasterio and CNR Clinical Physiology Institute, Via G. Moruzzi 1, 56124 Pisa, Italy.

E-mail: dneglia@ifc.cnr.it

Published online Sep. 19, 2011.

COPYRIGHT @ 2011 by the Society of Nuclear Medicine, Inc.
}

Key Words: myocardial blood flow reserve; coronary wall structure; coronary calcium; multislice computed tomography; positron emission tomography

J Nucl Med 2011; 52:1704-1712

DOI: 10.2967/jnumed.111.091009

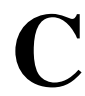
oronary blood flow reserve is a recognized indicator of the functional significance of coronary stenoses ( 1 ) and used to establish the need for coronary revascularization in the case of intermediate lesions (2). However, the use of absolute measurement of myocardial blood flow (MBF) and MBF reserve by PET has challenged this concept (3). Although PET-derived regional MBF reserve is inversely related to the degree of coronary stenoses, considerable interindividual variability exists $(4,5)$. Early atherosclerosis (6), the function of downstream coronary microcirculation (7), and cardiovascular risk burden (8) have been reported as additional determinants of MBF reserve. However, the relationship between all these components and MBF impairment remains undetermined, mainly because of the limitations of diagnostic approaches used to date.

Multislice CT (MSCT) and PET have the potential to better elucidate these relationships. MSCT can provide information not only on luminal narrowing but also on structural abnormalities of the vascular wall by means of densitometric analysis (9). Furthermore, MSCT can estimate coronary calcium content, an established indicator of atherosclerotic burden (10) and coronary risk (11) and a determinant of MBF reserve (12). Finally, the densitometric analysis of MSCT can also provide information regarding the fibroadipose and fibromuscular components of the coronary arterial wall, which may indicate early atherosclerotic damage (9). On the other hand, PET permits delineation of MBF reserve in the same patient and with the same image format. Thus, combined analysis of these 2 image datasets may provide direct assessment of the correlation between the structure of the coronary artery and MBF regulation in the corresponding downstream myocardial bed (13). 
Thus, we undertook this study to assess the relationship between coronary luminal narrowing, coronary wall components, cardiovascular risk factors, and regional and global MBF reserve.

\section{MATERIALS AND METHODS}

\section{Patient Population}

We studied 68 patients referred for noninvasive cardiac imaging at our institute because of anginalike chest pain and an intermediate (20\%-90\%) pretest likelihood of coronary artery disease (CAD) according to the Diamond and Forrester risk score. Twenty of these patients were enrolled in the EVINCI multicenter study (Evaluation of Integrated Cardiac Imaging in Ischemic Heart Disease), whereas 5 were enrolled in the ARTreat multicenter study (multilevel patient-specific artery and atherogenesis model for outcome prediction, decision support treatment, and virtual hands-on training). Patients underwent a myocardial perfusion PET scan at rest and under dipyridamole, followed by MSCT coronary angiography. Those with at least 1 coronary stenosis of $50 \%$ or greater underwent invasive coronary angiography (ICA). Exclusion criteria were previous percutaneous coronary interventions or bypass surgery, prior myocardial infarction, valvular heart disease, left ventricular (LV) dysfunction (LV ejection fraction $<45 \%$ ), contraindication to radiographic contrast medium, atrial fibrillation, and systemic diseases affecting midterm prognosis. The following cardiovascular risk factors were recorded for each patient: age, sex, diabetes mellitus, arterial hypertension, hypercholesterolemia, low high-density-lipoprotein cholesterol, smoking habit, and obesity. Accordingly, the Framingham risk score was determined as an indicator of integrated cardiovascular risk (14).

All patients were clinically stable before, during, and between the PET and MSCT angiography studies. The study protocol was approved by the local ethics committee; all patients signed an informed consent form.

\section{PET and MSCT Studies}

Patients were asked to fast overnight, avoid caffeine-containing foods, and withdraw $\beta$-blockers in the $24 \mathrm{~h}$ preceding the PET study (performed on a Discovery PET/CT scanner; GE Healthcare). Each acquisition was preceded by a CT transmission scan for photon attenuation correction (15). ${ }^{13} \mathrm{~N}$-ammonia was administered intravenously at a dose of $7.4 \mathrm{MBq} / \mathrm{kg}$ of body weight over a 10 - to 20 -s period. Twenty-eight dynamic frames were acquired over $8 \min$ (16 frames for $3 \mathrm{~s}, 11$ frames for $12 \mathrm{~s}$, and 1 frame for 300 s). Fifty minutes after the baseline study, another CT transmission scan was obtained, and dipyridamole was administered intravenously $(0.56 \mathrm{mg} / \mathrm{kg}$ of body weight over $4 \mathrm{~min})$. The dynamic ${ }^{13} \mathrm{~N}$-ammonia scan was started 2 min after the completion of the dipyridamole infusion. Two to $3 \mathrm{~min}$ after ${ }^{13} \mathrm{~N}$-ammonia injection, dipyridamole was antagonized by intravenous aminophylline in all patients. A 3-lead electrocardiogram was continuously monitored, and a 9-lead electrocardiogram and arterial blood pressure were obtained during ${ }^{13} \mathrm{~N}$-ammonia injection at rest, during dipyridamole infusion, and in the recovery phase. The whole PET protocol allowed us to limit mean radiation exposure to $3 \mathrm{mSv}$.

Within 1-6 wk from the completion of each PET study (20 \pm $18 \mathrm{~d}$ ), patients underwent 64-slice MSCT coronary angiography (LightSpeed VCT 64; GE Healthcare) with the following scan parameters: retrospective electrocardiogram gating, 912 channel detectors along the gantry and 64 channel detectors along the $z$-axis, tube voltage of $120 \mathrm{kV}$, tube current of $350-750 \mathrm{~mA}$ (depending on patient size), a scan field of view of $50 \mathrm{~cm}$, gantry rotation of $0.35 \mathrm{~s} /$ rotation, a matrix of $512 \times 512$, a slice thickness of $0.625 \mathrm{~mm}$, and a range of helical pitch of $0.18-0.24$. When appropriate, metoprolol (up to $5 \mathrm{mg}$ intravenously) - to lower the heart rate below 65 beats per minute-and isosorbide dinitrate (up to $1 \mathrm{mg}$ intravenously) - to guarantee maximal epicardial vasodilatation - were administered. Nonionic iodinated contrast medium (Iomeprol 400; Bracco) was injected via a peripheral vein according to a triphasic protocol using a programmable injector (Nemoto Dual Shot Injector; Nemoto Kyorindo Co. Ltd.): first a rapid injection of contrast medium (from 5 to 8 $\mathrm{mL} / \mathrm{s})$ was administered; in the second phase, $10 \mathrm{~mL}$ of contrast at $1 \mathrm{~mL} / \mathrm{s}$ were injected simultaneously with $25 \mathrm{~mL}$ of saline at $2.5 \mathrm{~mL} / \mathrm{s}$; finally, a $35-\mathrm{mL}$ saline flush was administered at 4 $\mathrm{mL} / \mathrm{s}$ (maximal total volume of contrast medium, $110 \mathrm{~mL}$ ). To time the scan, a region of interest was placed in the right ventricular cavity to detect peak contrast enhancement. Scans were obtained during breath hold; patients were monitored continuously through single-lead electrocardiography. The scan parameters were programmed to limit radiation exposure to $15 \mathrm{mSv}$ on average. After the procedure, patients were infused with saline $(500 \mathrm{~mL})$ to prevent contrast-induced nephropathy and instructed to have their serum creatinine rechecked between 2 and $7 \mathrm{~d}$ after the examination.

\section{PET and MSCT Analysis}

MBF was calculated from PET images according to a previously validated method $(16,17)$. Segmental MBFs were first computed according to the standardized 17-segment LV model (18). Then, patients' MSCT angiography images were fused with the pertinent PET volumetric perfusion images using a dedicated software program (CardIQ Fusion; GE Healthcare) (Fig. 1) that allowed coregistration of MSCT and PET images by a semiautomatic approach. Each myocardial segment was assigned to the respective major coronary branch (left anterior descending, circumflex, right coronary artery) (13). MBF reserve in each perfusion territory and for the whole left ventricle was computed as the ratio of MBFs during hyperemia and at rest and considered depressed if less than 2. The summed stress scores (SSS), summed rest scores, and summed difference scores were calculated automatically for the left ventricle by dedicated software (Emory Cardiac Toolbox for PET/CT; GE Healthcare). An SSS greater than 4 was considered indicative of significant stress-induced regional ischemia (19).

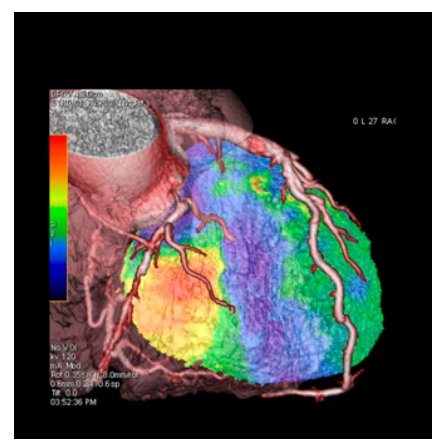

RGB

FIGURE 1. Fusion of MSCT coronary angiography image (left anterior descending visualization) with pertinent volumetric PET perfusion image. 
Transaxial MSCT images were reconstructed using a slice thickness of $0.625 \mathrm{~mm}$ and $0.4-\mathrm{mm}$ increments. The data were then transferred to a dedicated workstation (Advantage Workstation 4.3; GE Healthcare) for postprocessing. Lumen size (diameter and area) of the major coronary arteries was measured on Multiplanar Reformatting Images using an automatic interactive program to quantify coronary luminal narrowing. The severity of coronary stenoses was expressed with intervals of 5\%; a lumen reduction $50 \%$ or greater was considered a significant stenosis. In vessels showing multiple stenoses in series, only the most severe was considered. Volumetric analysis of the coronary vessel wall was evaluated using the Color Code Plaque analysis software (GE Healthcare) (9). This densitometric method, based on the Hounsfield scale, allows the computation of a cylindric volume around the vessel lumen to assess the relative contribution of calcium, fibroadipose, and fibromuscular components to the vessel wall. A similar algorithm has already been validated against intravascular ultrasound, the gold standard for the invasive assessment of vessel wall structure, using both 64-slice MSCT (20) and dual-source CT scanners (21). The algorithm of vessel wall analysis was calibrated in each patient according to densitometric parameters measured by a region of interest in the aortic root, LV myocardium, and epicardial fat. CT density less than 60 Hounsfield units (HUs) was attributed to a fibroadipose component and CT density between 60 and 200 HUs to a fibromuscular component, and calcified plaques were differentiated from the lumen when they had a density greater than 800 HUs using this system (Fig. 2). The program output gave a quantitative $\left(\mathrm{mm}^{3}\right)$ measurement of fibroadipose, fibromuscular, and calcium components for each main coronary vessel and for the entire coronary tree. Each component was expressed as percentage of vessel volume and used in per-vessel analyses. The percentage of each component in the whole coronary tree was used in the per-patient analyses.

\section{ICA}

Conventional ICA was performed using standard techniques and standard multiple projections. Coronary angiograms were quantified with dedicated computer software (Advanced Vessel Analysis, Innova 3DXR for Advanced Workstations; GE Healthcare) using an automatic edge-contour detection algorithm to compute stenosis severity expressed in percentage and coronary minimal luminal diameter. A coronary stenosis of $50 \%$ or greater was considered significant.

\section{Statistical Analysis}

Continuous variables were expressed as mean \pm SD and categoric variables as percentages. Simple regression analysis was used to correlate MSCT variables with regional MBF reserve and Framingham risk score. Groups were compared for categoric data using the Fisher exact test and for continuous variables using ANOVA followed by a Fisher protected least-significant difference test for multiple comparisons. The Kruskal-Wallis test, followed by the Dunn test, was used to analyze the prevalence of the coronary calcium component in the different vessel categories. Multiple logistic regression analysis was used to identify the variables significantly associated with depressed $(<2)$ regional and global MBF reserve. The predictive value of a variable was expressed as an odds ratio with corresponding 95\% confidence interval; the Wald test was used for significance. When volumetric characterization of vessel wall components was not technically feasible, nonanalyzable vessels and related patients were excluded from the regression analysis. All tests were 2 -sided. A $P$ value of less than 0.05 was considered statistically significant. All statistical analyses were performed using JMP statistical software (version 9.0; SAS Institute Inc.) and Stata statistical software (release 10; StataCorp. 2007).

\section{RESULTS}

\section{Patient-by-Patient Analysis}

The MSCT protocol was completed for every patient without significant adverse events. Mean heart rate before the procedure was 59 beats per minute (range, 46-74 beats per minute); $\beta$-blockers were administered in 15 patients. Presence and severity of coronary stenoses were evaluated in each of the 204 vessels analyzed; because of inadequate image quality in 8 vessels (5 patients), the volumetric characterization of vessel wall components was obtained in only 196 of 204 vessels (93\%). Coronary angiography by MSCT documented significant coronary stenoses ( $\geq 50 \%$ luminal diameter reduction) in at least 1 major coronary branch in 32 patients (47\%), nonsignificant stenoses $(<50 \%$ diameter reduction) in 15 patients $(22 \%)$, and normal coronary arteries in 21 patients $(31 \%)$. In the 32 patients with significant coronary stenoses, the lesions involved a single vessel in 16 patients, 2 vessels in 9 patients, and 3 vessels in 7 patients. ICA confirmed significant stenoses

FIGURE 2. Densitometric analysis of coronary wall structure based on Hounsfield scale; calcium, fibroadipose, and fibromuscular components. $\mathrm{CC}=$ calcium component; $F A C=$ fibroadipose component; $\mathrm{FMC}=$ fibromuscular component.

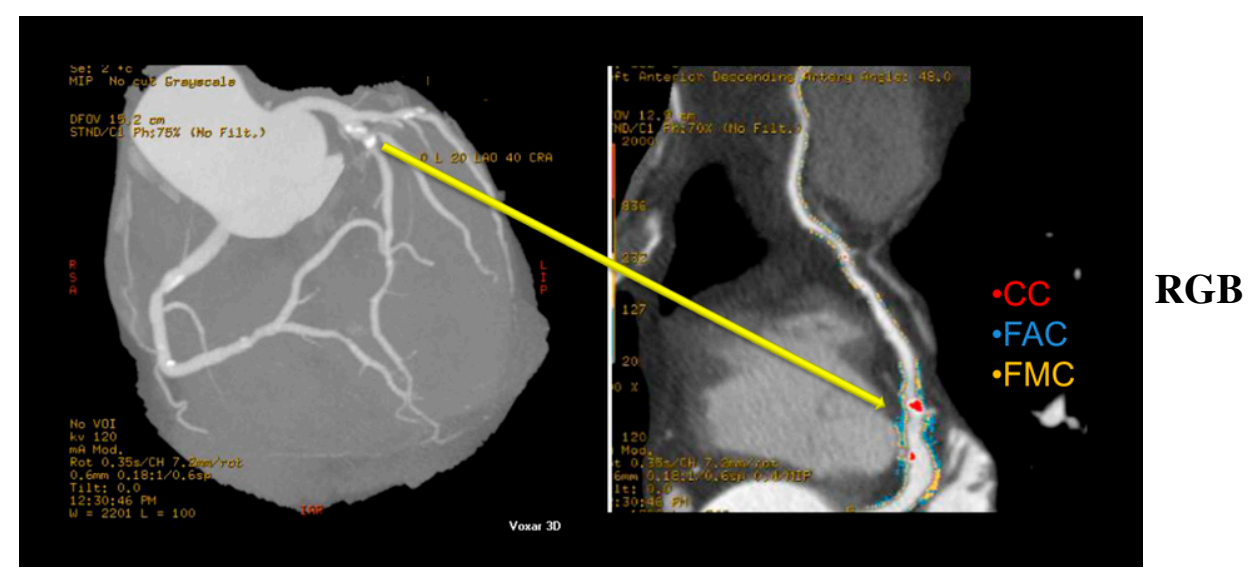


TABLE 1

Characteristics of Patients

\begin{tabular}{|c|c|c|c|c|c|}
\hline Parameter & $\begin{array}{l}\text { Overall } \\
(n=63)\end{array}$ & $\begin{array}{c}\text { Normal arteries } \\
\quad(n=21)\end{array}$ & $\begin{array}{l}<50 \% \text { stenosis } \\
\quad(n=15)\end{array}$ & $\begin{array}{l}\geq 50 \% \text { stenosis } \\
(n=27)\end{array}$ & $P$ \\
\hline Age $(y)$ & $62 \pm 11$ & $56 \pm 9$ & $62 \pm 12$ & $66 \pm 11^{*}$ & 0.014 \\
\hline Male sex & $37(59)$ & $10(48)$ & $10(67)$ & $17(63)$ & 0.436 \\
\hline Typical angina & $44(70)$ & $12(57)$ & $11(73)$ & $21(77)$ & 0.286 \\
\hline Pretest probability of CAD (\%) & $58.4 \pm 27.0$ & $45.6 \pm 28.5$ & $54.2 \pm 27.0^{\dagger}$ & $65.3 \pm 20.4^{\star}$ & 0.022 \\
\hline Family history of coronary heart disease & $17(30)$ & $6(29)$ & $4(27)$ & $7(26)$ & 0.978 \\
\hline Diabetes mellitus & $11(17)$ & $3(14)$ & $2(13)$ & $6(22)$ & 0.787 \\
\hline Hypercholesterolemia & $28(44)$ & $12(57)$ & $5(33)$ & $11(41)$ & 0.321 \\
\hline Low high-density-lipoprotein cholesterol & $27(43)$ & $7(33)$ & $7(47)$ & $13(48)$ & 0.545 \\
\hline Hypertension & $24(38)$ & $5(24)$ & $7(47)$ & $12(44)$ & 0.253 \\
\hline Obesity & $9(14)$ & $2(10)$ & $5(33)$ & $2(7)$ & 0.056 \\
\hline Smoking & $16(25)$ & $7(33)$ & $1(7)^{\dagger}$ & $8(29)$ & 0.155 \\
\hline Framingham risk score (\%) & $14.1 \pm 11.3$ & $10.4 \pm 6.3$ & $12.9 \pm 10.1$ & $17.7 \pm 13.6^{\dagger}$ & 0.091 \\
\hline LV ejection fraction (\%) & $57 \pm 11$ & $59 \pm 13$ & $55 \pm 12$ & $56 \pm 9$ & 0.514 \\
\hline LV end-diastolic volume $(\mathrm{mL})$ & $136 \pm 36$ & $133 \pm 35$ & $139 \pm 42$ & $136 \pm 34$ & 0.902 \\
\hline LV end-systolic volume (mL) & $58 \pm 30$ & $55 \pm 37$ & $56 \pm 22$ & $62 \pm 26$ & 0.774 \\
\hline Summed rest score & $5.9 \pm 7.5$ & $4.3 \pm 5.0$ & $6.3 \pm 8.2$ & $7.1 \pm 8.6$ & 0.483 \\
\hline Summed stress score & $8.2 \pm 8.1$ & $6.2 \pm 6.6$ & $7.6 \pm 8.4$ & $10.7 \pm 9.2$ & 0.186 \\
\hline Summed stress score $>4$ & $35(56)$ & $7(33)$ & $8(53)$ & $20(74)^{\dagger}$ & 0.019 \\
\hline Summed difference score & $2.5 \pm 3.3$ & $1.9 \pm 2.9$ & $1.4 \pm 2.6$ & $3.6 \pm 3.8$ & 0.079 \\
\hline MBF reserve & $2.0 \pm 0.5$ & $2.2 \pm 0.5$ & $2.1 \pm 0.6$ & $1.8 \pm 0.5^{\dagger}$ & 0.044 \\
\hline MBF reserve $<2$ & $33(52)$ & $6(29)$ & $9(60)$ & $18(66)^{\star}$ & 0.025 \\
\hline$\beta$-blockers & $21(33)$ & $6(29)$ & $4(27)$ & $11(41)$ & 0.554 \\
\hline Calcium channel blockers & $11(17)$ & $3(14)$ & $2(13)$ & $6(22)$ & 0.687 \\
\hline Nitrates & $14(22)$ & $1(5)$ & $5(33)^{\dagger}$ & $8(30)^{\dagger}$ & 0.059 \\
\hline Diuretics & $6(9)$ & $2(9)$ & $3(20)$ & $1(4)$ & 0.226 \\
\hline $\begin{array}{l}\text { Angiotensin-converting enzyme inhibitors } \\
\text { or angiotensin receptor blockers }\end{array}$ & $22(35)$ & 7 (33) & $4(27)$ & $11(41)$ & 0.645 \\
\hline Statins or fibrates $(n)$ & $21(33)$ & $4(19)$ & $5(33)$ & $12(44)^{\dagger}$ & 0.180 \\
\hline Aspirin & $25(40)$ & $8(38)$ & $6(60)$ & $13(48)$ & 0.758 \\
\hline \multicolumn{6}{|l|}{$\begin{array}{l}{ }^{{ }^{*} P}<0.01 \text { vs. normal. } \\
{ }^{\dagger} P<0.05 \text { vs. normal. } \\
\text { Data are mean }+ \text { SD or } n\end{array}$} \\
\hline
\end{tabular}

detected at MSCT in 27 of 32 patients (84\%) and in 48 of 55 vessels (87\%). Accordingly, 5 of 32 patients and 7 of 55 vessels were excluded from further analysis.

The demographic, clinical, and PET data of the study patients are summarized in Table 1. Patients with coronary stenosis (either nonsignificant or significant) were older, had a higher pretest probability of $\mathrm{CAD}$, and more frequently had inducible ischemia (SSS $>4$ ) and a reduced MBF reserve than patients with normal coronary arteries. Among patients with coronary stenoses, the presence of significant lesions did not determine significant differences for any of the variables explored.

\section{Vessel Structure and Regional MBF Reserve}

Of the 197 coronary arteries analyzed, 48 (25\%) showed a significant stenosis and 38 (19\%) a nonsignificant stenosis, whereas $111(56 \%)$ did not show any stenosis. Regional MBF reserve was significantly different in the territories perfused by normal coronary arteries $(2.13 \pm 0.54)$ from that in areas of nonsignificant $(1.91 \pm 0.58)$ and significant coronary stenosis $(1.63 \pm 0.51, P<0.001)$. Similarly, the calcium content was higher in the arteries with significant $(0.95 \% \pm 1.08 \%)$ or nonsignificant stenoses $(0.73 \% \pm$
$0.93 \%)$ than in those without stenoses $(0.11 \% \pm 0.38 \%$, $P<0.001)$. Conversely, the fibroadipose and fibromuscular components did not significantly differ between the various vessel categories (Fig. 3).

\section{Determinants of Regional MBF Reserve}

Regional MBF reserve was negatively correlated with the severity of coronary stenosis at MSCT $(R=-0.36)$ (Fig. 4). In patients in whom quantitative ICA data were available, the correlation between regional MBF reserve and stenosis severity was confirmed (Supplemental Fig. 1; supplemental materials are available online only at http://jnm. snmjournals.org). Regional MBF reserve was also negatively correlated with coronary calcium content $(R=-0.32)$ and the coronary fibroadipose component $(R=-0.20)$ (Fig. 4) but not with the fibromuscular component. On multiple logistic regression analysis, significant coronary stenoses $(P<$ $0.047)$ and coronary calcium content $(P<0.017)$ remained the only independent determinants of regional MBF reserve (Table 2), because of the internal correlation between coronary calcium and fibroadipose content $(R=0.25, P=0.01)$. Interestingly, the fibroadipose component of the arterial wall 
FIGURE 3. Regional MBF reserve and relative prevalence of coronary calcium, fibroadipose, and fibromuscular components in different vessel categories. $\mathrm{CC}=$ calcium component; FAC = fibroadipose component; FMC = fibromuscular component.
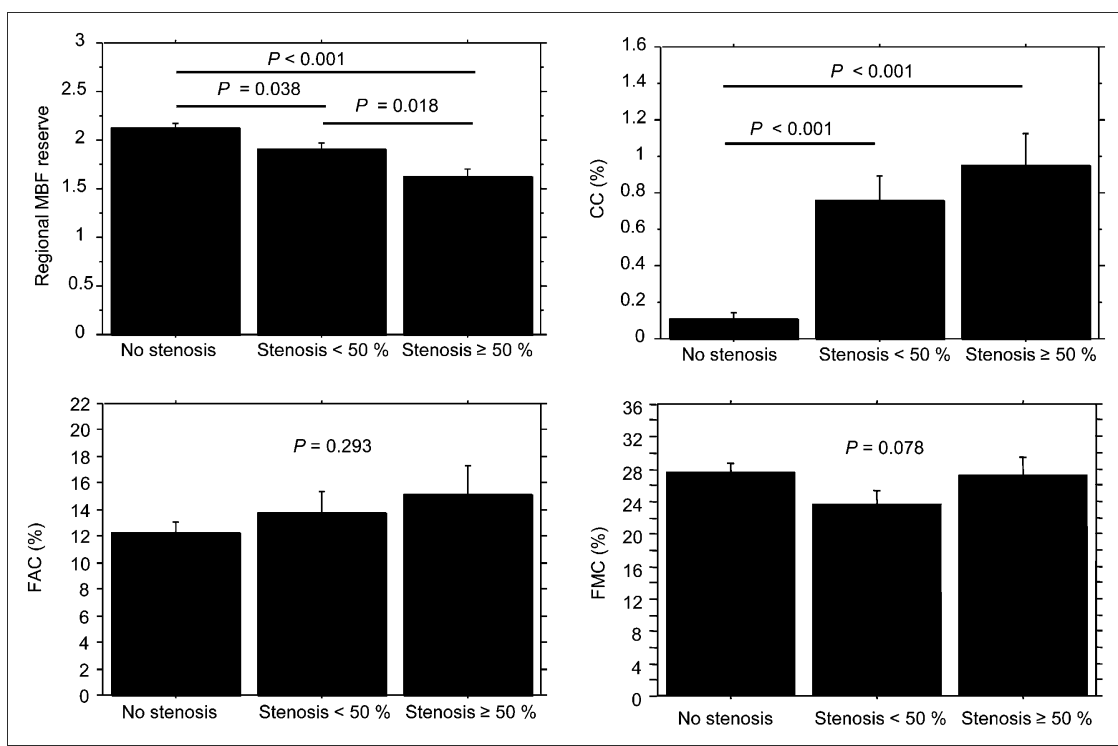

was a determinant of MBF reserve only in the presence of coronary stenoses (Fig. 5A).

\section{Correlation Between Atherosclerotic Burden and MBF Reserve}

MBF reserve was markedly heterogeneous (range, 1.243.61) in regions perfused by vessels without a significant stenosis. This variability was largely explained by the atherosclerotic burden of each vessel, estimated by the degree of coronary calcium content. In fact, a correlation was found between the amount of coronary calcium content and downstream MBF reserve in vessels without a significant stenosis $(R=-0.28, P=0.006)$ but not downstream from significantly stenotic vessels $(R=-0.20, P=0.10)$ (Fig. $5 B)$.

\section{Determinants of Global MBF Reserve}

Global coronary calcium content was an independent determinant of reduced global MBF reserve after adjustment for major cardiovascular risk factors, LV ejection fraction, and other MSCT parameters using multivariate analysis $(P=0.045$, Table 3$)$. However, when the Framingham risk score, an indicator of integrated cardiovascular risk, was used, it was the only significant determinant of global MBF reserve (odds ratio, 1.18 [95\% confidence interval, 1.02-1.37], $P=0.028$ ), overwhelming the effect of MSCT variables in the predictive model. Interestingly, the Framingham risk score was significantly correlated with the fibroadipose component $(R=0.43)$ and calcium content $(R=0.41)$ of the whole coronary tree but not with the presence and number of significant coronary stenoses.
FIGURE 4. Stenosis severity, coronary calcium component, and coronary fibroadipose component vs. downstream regional MBF reserve. $\mathrm{CC}=$ calcium component; $\mathrm{FAC}=$ fibroadipose component.
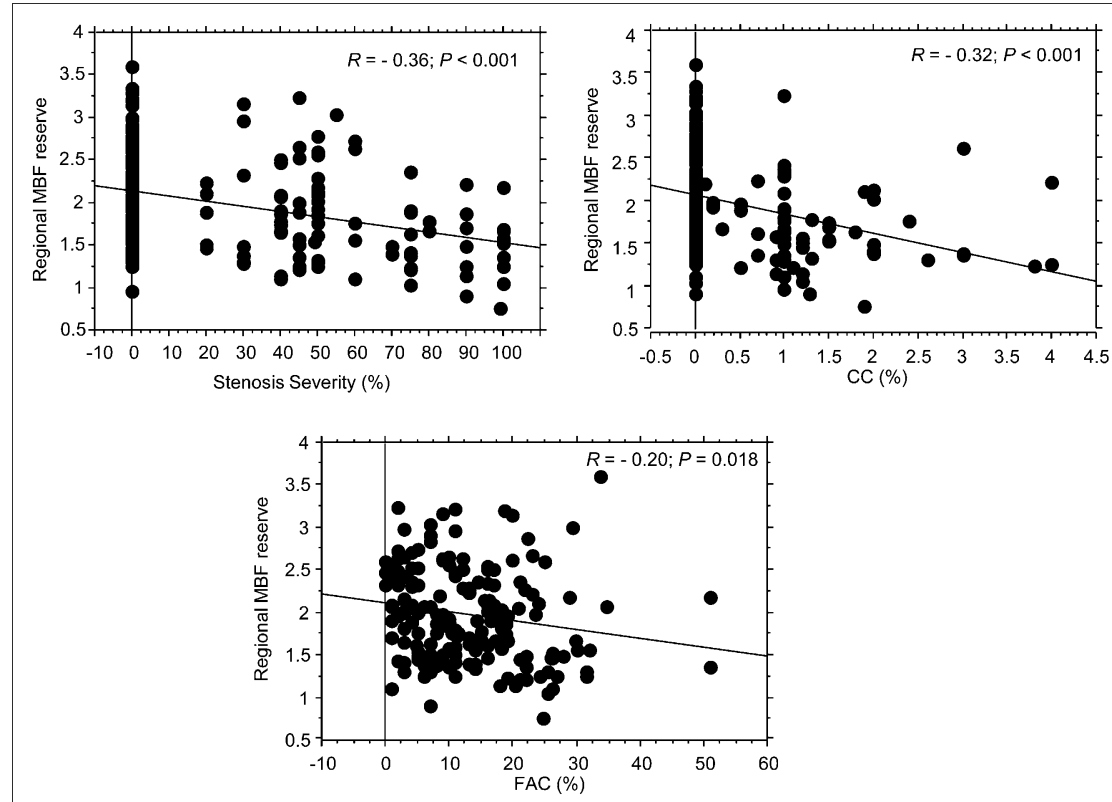
TABLE 2

Determinants of Depressed Regional MBF Reserve

\begin{tabular}{|c|c|c|c|c|c|c|}
\hline \multirow[b]{3}{*}{ Variable } & \multicolumn{6}{|c|}{ Logistic regression } \\
\hline & \multicolumn{3}{|c|}{ Univariate } & \multicolumn{3}{|c|}{ Multivariate } \\
\hline & Odds ratio & $95 \%$ confidence interval & $P$ & Odds ratio & 95\% confidence interval & $P$ \\
\hline Stenosis $\geq 50 \%, 0-1$ & 2.98 & $1.49-5.97$ & 0.002 & 2.11 & $1.01-4.46$ & 0.047 \\
\hline Calcium component (\%) & 2.10 & $1.32-3.27$ & 0.001 & 1.80 & $1.11-2.92$ & 0.017 \\
\hline Fibroadipose component (\%) & 1.03 & $1.01-1.08$ & 0.046 & 1.02 & $0.98-1.07$ & 0.214 \\
\hline Fibromuscular component (\%) & 0.98 & $0.95-1.01$ & 0.203 & 0.99 & $0.96-1.02$ & 0.471 \\
\hline
\end{tabular}

\section{DISCUSSION}

This study shows that an abnormal coronary wall structure affects regional MBF reserve beyond the presence and severity of coronary stenoses. Specifically, calcium content was the main determinant of impaired regional MBF reserve, whereas the fibroadipose component of the vessel wall was related with MBF reserve only in the presence of coronary stenoses. Interestingly, both calcium and fibroadipose components of the whole coronary tree were significantly related to individual cardiovascular risk burden. Accordingly, the Framingham risk score was the only independent determinant of global MBF reserve.

\section{Determinants of Regional MBF Reserve: Coronary Stenosis and Vessel Structure}

The relationship between coronary stenosis and regional MBF reserve was first documented by Gould and Lipscomb (1). This original concept, although validated in humans using angiographic estimates of stenosis severity and measurement of MBF reserve by PET (4), has been recently questioned (3). In fact, PET studies have shown an unexpected variability of downstream MBF reserve in vessels that were mildly stenotic and even in angiographically normal coronary arteries (5). Moreover, even fractional flow reserve, considered the reference method for assessing the functional significance of coronary stenoses, correlates poorly with the degree of luminal narrowing measured by invasive angiography or MSCT $(22,23)$. Possible interpretations include the inaccuracy of angiographic measures of coronary stenoses, an abnormal MBF regulation $(24,25)$, endothelial dysfunction (26), the effect of diffuse atherosclerosis on coronary flow (27), or structural and functional abnormalities of coronary microcirculation (8).

In the present study, although MBF reserve was negatively related to the degree of coronary stenoses, individual data were rather dispersed, mainly downstream from nonsignificant stenoses or in normal vessels. This variability was partly explained by coronary calcium content. Within the wall of vessels without significant stenoses, calcium content may express the presence of early atherosclerosis, which in turn impairs downstream myocardial perfusion (26). A similar correlation has been reported by Curillova et al. (12), although the presence and extent of coronary stenoses was not directly evaluated in that study
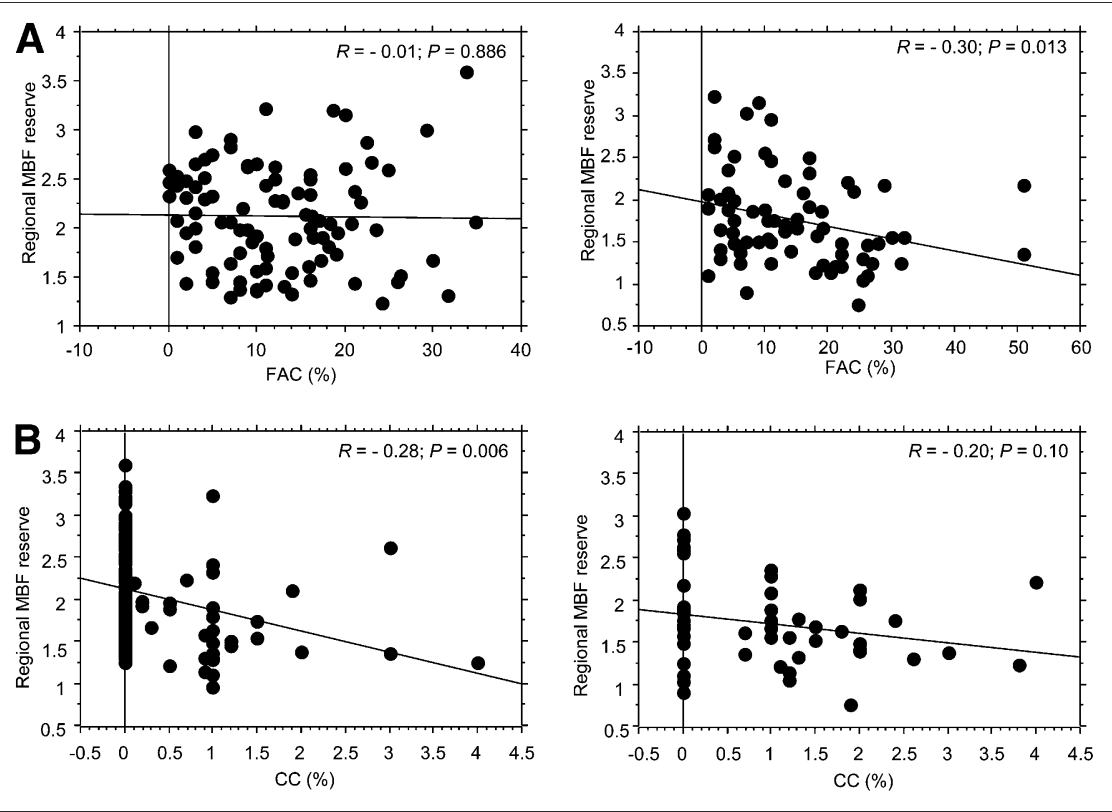

FIGURE 5. (A) Coronary fibroadipose component vs. regional MBF reserve in coronary arteries without stenoses (left) and coronary arteries with stenoses (right). (B) Coronary calcium component vs. downstream regional MBF reserve in vessels that were normal or without significant stenoses (left) and vessels with significant stenoses (right). $\mathrm{CC}=$ calcium component; FAC = fibroadipose component. 
TABLE 3

Determinants of Depressed Global MBF Reserve

\begin{tabular}{|c|c|c|c|c|c|c|}
\hline \multirow[b]{3}{*}{ Variable } & \multicolumn{6}{|c|}{ Logistic regression } \\
\hline & \multicolumn{3}{|c|}{ Univariate } & \multicolumn{3}{|c|}{ Multivariate } \\
\hline & Odds ratio & $95 \%$ confidence interval & $P$ & Odds ratio & $95 \%$ confidence interval & $P$ \\
\hline Age $(n)$ & 1.02 & $0.97-1.06$ & 0.498 & 1.04 & $0.85-1.03$ & 0.529 \\
\hline Sex (male) & 1.38 & $0.52-3.64$ & 0.521 & 3.69 & $0.93-1.16$ & 0.233 \\
\hline Family history of CAD, 1-0 & 0.64 & $0.22-1.82$ & 0.399 & 1.58 & $0.24-10.19$ & 0.633 \\
\hline Smoking, 1-0 & 1.37 & $0.45-4.17$ & 0.576 & 4.81 & $0.32-22.67$ & 0.257 \\
\hline Diabetes mellitus, 1-0 & 2.00 & $0.54-7.41$ & 0.299 & 1.11 & $0.09-2.89$ & 0.084 \\
\hline Hypercholesterolemia, 1-0 & 1.86 & $0.71-4.91$ & 0.209 & 2.13 & $0.74-6.19$ & 0.164 \\
\hline $\begin{array}{l}\text { Low high-density-lipoprotein } \\
\text { cholesterol, } 1-0\end{array}$ & 2.19 & $0.81-5.96$ & 0.126 & 2.17 & $0.78-6.21$ & 0.071 \\
\hline Hypertension, 1-0 & 1.76 & $0.65-5.76$ & 0.266 & 0.56 & $0.20-12.39$ & 0.676 \\
\hline Obesity (body mass index $>30$ ), $1-0$ & 0.96 & $0.83-1.09$ & 0.475 & 0.97 & $0.73-1.27$ & 0.799 \\
\hline Left ventricular EF, (\%) & 0.98 & $0.94-1.04$ & 0.589 & 1.02 & $0.92-1.12$ & 0.765 \\
\hline Significant coronary stenosis $(n)$ & 1.73 & $1.02-2.92$ & 0.040 & 2.79 & $0.79-9.81$ & 0.111 \\
\hline Global calcium component (\%) & 2.33 & $1.03-5.26$ & 0.041 & 2.34 & $1.01-5.39$ & 0.045 \\
\hline Global fibroadipose component (\%) & 1.04 & $0.98-1.11$ & 0.188 & 0.99 & $0.89-1.12$ & 0.949 \\
\hline Global fibromuscular component (\%) & 0.97 & $0.93-1.03$ & 0.399 & 0.95 & $0.84-1.07$ & 0.384 \\
\hline
\end{tabular}

and, as a result, the relative contribution of abnormal wall structure and luminal narrowing could not be established.

An additional novel finding of our study was that a higher fibroadipose component of the coronary wall was correlated with a reduction of downstream MBF reserve in the presence of luminal coronary narrowing. The clinical and pathophysiologic relevance of this result remains unclear. An increased fibroadipose component might highlight functionally active coronary atherosclerosis (28) or indicate the presence of widespread atheroma along the entire coronary, a condition that may additively impair myocardial perfusion even in the presence of focal stenoses $(27,29)$.

\section{Determinants of Global MBF Reserve: Role of Cardiovascular Risk Factors}

It is widely accepted that cardiovascular risk factors can impair myocardial perfusion even in the absence of significant coronary stenosis $(30,31)$ and that globally depressed MBF reserve negatively affects patient outcome beyond the extent of coronary artery disease (32). Although the consecutive pathogenetic link between risk factors, stenotic coronary disease, and depressed MBF reserve is accepted, the mechanisms of a possible direct effect of these factors on coronary function are still debated.

In our study, coronary calcium content was the major determinant of depressed global MBF reserve after adjustment for individual cardiovascular risk factors, extent of coronary disease, and LV ejection fraction. However, when risk factors were integrated into the Framingham risk score, this indicator of global cardiovascular risk turned out to be the only significant predictor of depressed global MBF reserve, overwhelming the effect of coronary calcium. This observation extends to previous reports on the association between Framingham risk score and global MBF reserve in patients without coronary stenoses (8), showing that a higher cardiovascular risk burden remains an independent predictor of depressed global MBF reserve, even in patients with different degrees of coronary disease.

\section{Study Limitations}

This study has several limitations. Because of the relatively limited sample size and lack of an invasive assessment of coronary wall structure and function, as provided by intravascular ultrasound and fractional flow reserve (33), the accuracy of combined MSCT and PET in detecting different stages of CAD could not be evaluated. However, the main purpose of the study was not to optimize the diagnostic application of PET/CT, but rather to noninvasively assess the relationships between coronary stenosis, wall structure, and altered MBF reserve. In addition, calcium content was measured from MSCT angiography without evaluating the Agatston calcium score, thus reducing radiation exposure by about $10 \%$.

The presence of contrast within the lumen might have limited the ability to differentiate vessel wall components. Nevertheless, the selection of an appropriate window level allowed the differentiation of contrast inside the coronary lumen from fibroadipose ( $<60 \mathrm{HU}$ ), fibromuscular (60 to $200 \mathrm{HU}$ ), and calcium ( $>800 \mathrm{HU}$ ) components. Although the reduction of the window of $\mathrm{HU}$ for calcium recognition may have caused an underestimation of calcium volume (20), unpublished data from our laboratory (collected from 2008 to 2011) show a strong correlation between the present method and the Agatston score $(\mathrm{y}=1.47+$ $\left.0.128 \mathrm{x} ; R^{2}=0.874, \mathrm{~F}_{250}=660.6, P<0.0001\right)$.

Another limitation is that the presence of calcium may have limited the ability of MSCT in evaluating stenosis severity. In reality, the effect of calcium on MBF reserve could be demonstrated downstream from coronary arteries independently of the extent of detected luminal narrowing. 
In our study, only patients with at least 1 significant coronary stenosis underwent ICA. Thus, we were not able to test the accuracy of MSCT angiography in assessing the severity of nonsignificant lesions. However, all of the 14 nonsignificant stenoses demonstrated at MSCT for patients with a significant lesion in another coronary artery were confirmed by ICA.

Summed rest score and SSS showed a high interindividual variability, even in patients without significant coronary stenoses. Because of the elevated negative predictive value of MSCT (34), it is improbable that missed significant lesions might explain this result. On the other hand, a similarly high interindividual variability has been demonstrated in regional MBF reserve downstream from vessels without significant stenosis, suggesting a functional impairment of coronary macro- or microcirculation as a probable cause of both findings (35).

Finally, although we usually perform MSCT and PET within the same session for clinical purposes (thus allowing the PET examination to be avoided if MSCT is negative), we separated the 2 imaging modalities for research purposes. However, because treatment was unchanged and the imaging coregistration process was unaffected by this approach, we do not believe that results have been substantially influenced.

\section{CONCLUSION}

This study shows that regional MBF reserve is an integrated measurement of the anatomic and functional effects of coronary atherosclerosis on flow regulation. Both luminal stenosis and structural abnormalities of the coronary wall were associated with impaired downstream myocardial perfusion. The assessment of absolute MBF reserve by PET, integrated with the evaluation of coronary anatomy and structure by MSCT, provides overall information on the functional relevance of the atherosclerotic process.

\section{DISCLOSURE STATEMENT}

The costs of publication of this article were defrayed in part by the payment of page charges. Therefore, and solely to indicate this fact, this article is hereby marked "advertisement" in accordance with 18 USC section 1734.

\section{ACKNOWLEDGMENTS}

We thank Alison Frank for her invaluable work in editing the manuscript. This study was partly supported by a grant from the FP7-CP-FP 2007 project (grant agreement 222915, EVINCI) and a grant from the FP7-ICT-2007 project (grant agreement 224297, ARTreat). No other potential conflict of interest relevant to this article was reported.

\section{REFERENCES}

1. Gould KL, Lipscomb K. Effects of coronary stenoses on coronary flow reserve and resistance. Am J Cardiol. 1974;34:48-55.

2. Kern MJ, Lerman A, Bech JW, et al. Physiological assessment of coronary artery disease in the cardiac catheterization laboratory: a scientific statement from the
American Heart Association Committee on Diagnostic and Interventional Cardiac Catheterization, Council on Clinical Cardiology. Circulation. 2006;114:1321-1341.

3. Gould KL. Does coronary flow trump coronary anatomy? JACC Cardiovasc Imaging. 2009;2:1009-1023.

4. Uren NG, Melin JA, De Bruyne B, Wijns W, Baudhuin T, Camici PG. Relation between myocardial blood flow and the severity of coronary-artery stenosis. $N$ Engl J Med. 1994;330:1782-1788.

5. Anagnostopoulos C, Almonacid A, El Fakhri G, et al. Quantitative relationship between coronary vasodilator reserve assessed by $82 \mathrm{Rb}$ PET imaging and coronary artery stenosis severity. Eur J Nucl Med Mol Imaging. 2008;35: 1593-1601.

6. Verna E, Ceriani L, Giovanella L, Binaghi G, Garancini S. "False-positive" myocardial perfusion scintigraphy findings in patients with angiographically normal coronary arteries: insights from intravascular sonography studies. $\mathrm{J} \mathrm{Nucl}$ Med. 2000;41:1935-1940.

7. Camici PG, Crea F. Coronary microvascular dysfunction. N Engl J Med. 2007; 356:830-840.

8. Dorbala S, Hassan A, Hainonen T. Coronary vasodilator reserve and Framingham risk scores in subjects at risk for coronary artery disease. $J$ Nucl Cardiol. 2006;13:761-767.

9. Kunita E, Fujii T, Urabe Y, et al. Coronary plaque stabilization followed by color code plaque analysis with 64- slice multidetector row computed tomography. Circ J. 2009;73:772-775.

10. Schmermund A, Denktas AE, Rumberger JA, et al. Independent and incremental value of coronary artery calcium for predicting the extent of angiographic coronary artery disease: comparison with cardiac risk factors and radionuclide perfusion imaging. J Am Coll Cardiol. 1999;34:777-786.

11. Schenker MP, Dorbala S, Hong EC, et al. Interrelation of coronary calcification, myocardial ischemia, and outcomes in patients with intermediate likelihood of coronary artery disease: a combined positron emission tomography/computed tomography study. Circulation. 2008;117:1693-1700.

12. Curillova Z, Yaman BF, Dorbala S, et al. Quantitative relationship between coronary calcium content and coronary flow reserve as assessed by integrated PET/CT imaging. Eur J Nucl Med Mol Imaging. 2009;36:1603-1610.

13. Javadi MS, Lautamäki R, Merrill J, et al. Definition of vascular territories on myocardial perfusion images by integration with true coronary anatomy: a hybrid PET/CT analysis. J Nucl Med. 2010;51:198-203.

14. D'Agostino RB, Russell MW, Huse DM, et al. Primary and subsequent coronary risk appraisal: new results from the Framingham study. Am Heart J. 2000;139: 272-281.

15. Koepfli P, Hany TF, Wyss CA, et al. CT attenuation correction for myocardial perfusion quantification using a PET/CT hybrid scanner. J Nucl Med. 2004;45: 537-542.

16. Bellina CR, Parodi O, Camici P, et al. Simultaneous in vitro and in vivo validation of nitrogen-13-ammonia for the assessment of regional myocardial blood flow. J Nucl Med. 1990;31:1335-1343.

17. Nekolla SG, Miethaner C, Nguyen N, Ziegler SI, Schwaiger M. Reproducibility of polar map generation and assessment of defect severity and extent assessment in myocardial perfusion imaging using positron emission tomography. Eur $J$ Nucl Med. 1998;25:1313-1321.

18. Cerqueira MD, Weissman NJ, Dilsizian V, et al. Standardized myocardial segmentation and nomenclature for tomographic imaging of the heart: a statement for healthcare professionals from the Cardiac Imaging Committee of the Council on Clinical Cardiology of the American Heart Association. Circulation. 2002; 105:539-542.

19. van Werkhoven JM, Schuijf JD, Gaemperli O, et al. Prognostic value of multislice computed tomography and gated single-photon emission computed tomography in patients with suspected coronary artery disease. J Am Coll Cardiol. 2009;53:623-632.

20. Achenbach S, Moselewski F, Ropers D, et al. Detection of calcified and noncalcified coronary atherosclerotic plaque by contrast-enhanced, submillimeter multidetector spiral computed tomography: a segment-based comparison with intravascular ultrasound. Circulation. 2004;109:14-17.

21. Marwan M, Taher MA, El Meniawy K, et al. In vivo CT detection of lipid-rich coronary artery atherosclerotic plaques using quantitative histogram analysis: A head to head comparison with IVUS. Atherosclerosis. 2011;215:110-115.

22. Meijboom WB, Van Mieghem CA, van Pelt N, et al. Comprehensive assessment of coronary artery stenoses: computed tomography coronary angiography versus conventional coronary angiography and correlation with fractional flow reserve in patients with stable angina. J Am Coll Cardiol. 2008;52:636-643.

23. Wijpkema JS, Dorgelo J, Willems TP, et al. Discordance between anatomical and functional coronary stenosis severity. Neth Heart J. 2007;15:5-11.

24. Uren NG, Marraccini P, Gistri R, de Silva R, Camici PG. Altered coronary vasodilator reserve and metabolism in myocardium subtended by normal 
arteries in patients with coronary artery disease. J Am Coll Cardiol. 1993;22: 650-658.

25. Sambuceti G, Marzullo P, Giorgetti A, et al. Global alteration in perfusion response to increasing oxygen consumption in patients with single-vessel coronary artery disease. Circulation. 1994;90:1696-1705.

26. Zeiher AM, Drexler H, Wollschläger H, Just H. Endothelial dysfunction of the coronary microvasculature is associated with coronary blood flow regulation in patients with early atherosclerosis. Circulation. 1991;84:1984-1992.

27. Gould KL, Nakagawa Y, Nakagawa K, et al. Frequency and clinical implications of fluid dynamically significant diffuse coronary artery disease manifest as graded, longitudinal, base-to-apex myocardial perfusion abnormalities by non invasive positron emission tomography. Circulation. 2000;101:1931-1939.

28. Schindler TH, Nitzsche EU, Olschewski MJ, et al. PET-measured responses of MBF to cold pressor testing correlate with indices of coronary vasomotion on quantitative coronary angiography. J Nucl Med. 2004;45:419-428.

29. De Bruyne B, Hersbach F, Pijls NH, et al. Abnormal epicardial coronary resistance in patients with diffuse atherosclerosis but "normal" coronary angiography. Circulation. 2001;104:2401-2406.
30. Kaufmann PA, Gnecchi-Ruscone T, Schäfers KP, Lüscher TF, Camici PG. Low density lipoprotein cholesterol and coronary microvascular dysfunction in hypercholesterolemia. J Am Coll Cardiol. 2000;36:103-109.

31. Di Carli MF, Bianco-Batlles D, Landa ME, et al. Effects of autonomic neuropathy on coronary blood flow in patients with diabetes mellitus. Circulation. 1999; 100:813-819.

32. Tio RA, Dabeshlim A, Siebelink HM, et al. Comparison between the prognostic value of left ventricular function and myocardial perfusion reserve in patients with ischemic heart disease. J Nucl Med. 2009;50:214-219.

33. Kajander S, Joutsiniemi E, Saraste M, et al. Cardiac positron emission tomography/computed tomography imaging accurately detects anatomically and functionally significant coronary artery disease. Circulation. 2010;122:603-613.

34. Abdulla J, Abildstrom SZ, Gotzsche O, et al. 64-multislice detector computed tomography coronary angiography as potential alternative to conventional coronary angiography: a systematic review and meta-analysis. Eur Heart J. 2007;28:3042-3050.

35. Gimelli A, Rossi G, Landi P, et al. Stress/rest myocardial perfusion abnormalities by gated SPECT: still the best predictor of cardiac events in stable ischemic heart disease. J Nucl Med. 2009;50:546-553. 